\section{А. А. Чуклин}

Севастопольская служба научно-технической поддержки, ОП НТЦ НАЭК «Энергоатом», г. Севастополь, Украина

\section{Моделирование конвекции с использованием одномерных кодов}

Показана возможность физически корректного моделирования процессов тепломассопереноса в однофазных средах с помощью одномерных компьютерных кодов. Приведены рекомендации по выбору оптимальных нодализационных схем.

Ключевые слова: конвекция, компьютерное моделирование, одномерные коды, MELCOR.

\section{0. О. Чуклін}

\section{Моделювання конвекціїз використанням одновимірніх} кодів

Описано можливість фізично коректного моделювання процесів тепломасоперенесення в однофазних середовищах за допомогою одновимірних комп'ютерних кодів. Наведено рекомендації з вибору оптимальних нодалізаційних схем.

Ключов і слов а: конвекція, комп'ютерне моделювання, одновимірні коди, MELCOR.
$\mathrm{P}$ ассматриваемый в данной статье компьютерный код MELCOR является одномерным с «сосредоточенными» параметрами. Параметры по всему моделируемому контрольному объему эквивалентны друг другу, что значительно усложняет моделирование трехмерных систем. Существенная проблема, возникающая при моделировании неодномерных процессов тепломассообмена и гидродинамики, - учет рециркуляции, влияющей на их динамику.

Рециркуляция в объемах связана с возникновением конвективного перемешивания. Конвекция - это явление переноса теплоты в жидкостях, газах или сыпучих средах потоками вещества за счет переноса массы. Естественная конвекция возникает в веществе самопроизвольно при его неравномерном нагревании в поле тяготения.

Для создания квазитрехмерных моделей приходится прибегать к более детальной нодализации моделируемых элементов. Одна из задач, стоящих перед разработчиком компьютерной модели энергоустановки, заключается в выборе оптимального количества элементов нодализационной схемы.

В случае упрощенного моделирования программа может не учесть некоторые физические процессы, в частности:

конвекцию (разогрев недогретого теплоносистеля в компенсаторе давления и т. п.);

перетоки сред между участками моделируемого объекта (например, верхняя камера смешения реактора, заданная одним контрольным объемом, не позволит учесть перетоки теплоносителя при отключении одного из ГЦН);

температурные изменения по длине и высоте объекта (например, разбивка трубчатки парогенератора на участки по длине и высоте).

С другой стороны, использование неоправданно детализированной нодализации приведет как к увеличению расчетного времени, так и к накоплению вычислительной погрешности [1].

Для рассмотрения задачи учета конвекции одномерными кодами сравним результаты стендового эксперимента с результатами расчетного эксперимента, выполненного с использованием системы дифференциальных уравнений, а также с результатами расчетного эксперимента, выполненного с использованием компьютерного кода MELCOR.

Стендовый эксперимент. Экспериментальный стенд представляет собой заполненную водой металлическую емкость, к одной из стенок которой подводится тепло от нагретой до $100{ }^{\circ} \mathrm{C}$ жидкости, которая, в свою очередь, получает тепло от трубчатого электронагревателя мощностью 2000 Вт. В качестве измерительных приборов используются видеорегистратор, двухмерная измерительная плоскость и термопары (ТП), расположенные согласно схеме, изображенной на рис. 1. Для визуализации поля скоростей в емкость вводится краситель - раствор фукорцина (лат. fucorcinum, также жидкость Кастеллани или краска Кастеллани), который обладает характерным яркомалиновым цветом.

В процессе эксперимента вблизи греющей стенки наблюдается устойчивый поток жидкости, устремленный вверх. Толщина слоя находится в пределах 0,019 м, скорость подъема слоя $-0,05$ м/с. Движение жидкости в верхней части объема происходит в направлении «от» греющей стенки. Скорость и толщина слоя жидкости, движущейся вдоль поверхности, изменяются со временем в зависимости от отдаленности потока от греющей стенки. Средняя скорость потока на поверхности составляет $0,027 \mathrm{~m} / \mathrm{c}$. Ввиду отсутствия «холодного» источника (на противоположной от греющей стенки стороне), движение слоя жидкости 


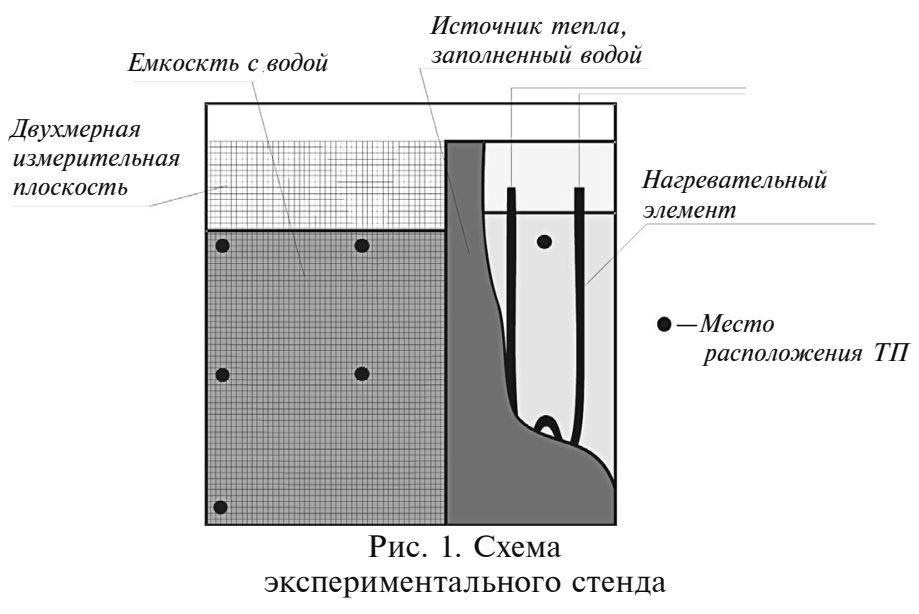

вдоль стенки вниз (подобно слою жидкости, движущемуся вверх вблизи «горячей» стенки) почти не происходит Согласно показаниям термопар, в верхней части объема выделяется зона с нагретой жидкостью, толщина которой с течением времени увеличивается. Начинается медленное закручивание всего нагретого потока (рис. 2).

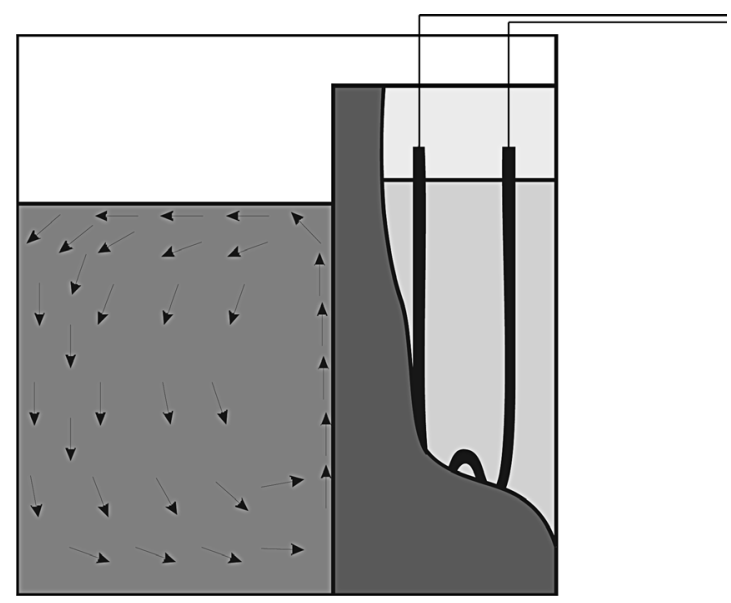

Рис. 2. Графическое изображение результатов стендового эксперимента

Поскольку «холодный» источник отсутствует, процесс является нестационарным: в связи с постепенным нагреванием жидкости изменяются поля температур и скоростей. Для сравнения результатов стендового эксперимента с результатами расчетов рассматривается интервал времени от начала введения «горячего» источника в систему до повторного достижения «языком» нагретой жидкости греющей стенки.

Расчетный эксперимент с использованием математической модели. Для расчетного эксперимента создана математическая модель экспериментальной установки. Наиболее популярной моделью для описания конвекции в жидкостях и газах являются уравнения тепловой конвекции в приближении Буссинеска - Обербека (A. Oberbeck, 1879; J. Boussinesq, 1903). Модель включает уравнение Навье - Стокса, уравнение теплопроводности и уравнение несжимаемости. Основная идея приближения состоит в особенности учета зависимости плотности от температуры [2].

Примем, что характер течения жидкости в рассматриваемом объеме одинаков во всех вертикальных плоскостях, перпендикулярных к греющей поверхности. В таком случае для исследования кинематики и динамики потока достаточно рассмотреть плоскую задачу, т. е. когда скорость и давление жидкости зависят только от двух координат $(x ; y)$.

Уравнением движения, описывающим изменение скорости во времени и пространстве, является уравнение Навье - Стокса [2], которое в переменных «скорость давление» имеет следующий вид:

$$
\frac{d w}{d \tau}+(w \nabla) w=-\frac{\nabla p}{\rho}+\mu \nabla w,
$$

где $w$ - скорость; $\tau$ - время; $\rho$ - плотность; $p$ - давление; $\mu-$ динамический коэффициент вязкости; $\Delta-$ оператор Лапласа; $\nabla$ - оператор Набла.

Уравнение неразрывности для несжимаемой жидкости в двухмерной задаче имеет вид

$$
\frac{\partial w_{x}}{\partial x}+\frac{\partial w_{y}}{\partial y}=0
$$

где $w_{x}-$ проекция скорости на координатную ось $x ; w_{y}-$ проекция скорости на координатную ось $y ; y-y$-координата; $x-x$-координата.

Чтобы сделать систему уравнений замкнутой, необходимо добавить дифференциальное уравнение, описывающее температурное поле в движущейся жидкости, т. е. уравнение энергии. При записи дифференциального уравнения энергии принимается, что жидкость однородна и изотропна, энергия деформации мала по сравнению с изменением внутренней энергии. Уравнение энергии имеет вид [3]

$$
\frac{\partial t}{\partial \tau}+w_{x} \frac{\partial t}{\partial x}+w_{y} \frac{\partial t}{\partial y}=-a\left(\frac{\partial^{2} t}{\partial x^{2}}+\frac{\partial^{2} t}{\partial y^{2}}\right),
$$

где $t$ - температура; $a-$ коэффициент теплопроводности.

Для моделирования плоских течений широко используются переменные «функция тока - вихрь скорости» вместо физических (естественных) переменных «скорость - давление» $[4,5]$. Проекции скоростей на координатные оси $x$ и $y$, представленные через функцию тока $\psi$, имеют вид

$$
w_{x}=\frac{\partial \psi}{\partial y} ; w_{y}=-\frac{\partial \psi}{\partial x}
$$

где $\psi-$ функция тока.

Вихрь скорости двухмерных течений представлен в виде

$$
\omega=\frac{\partial w_{x}}{\partial x}-\frac{\partial w_{y}}{\partial y}
$$

где $\omega-$ вихрь скорости.

Приняв во внимание (4), получим уравнение [5, 7]

$$
\Delta \psi=-\omega .
$$




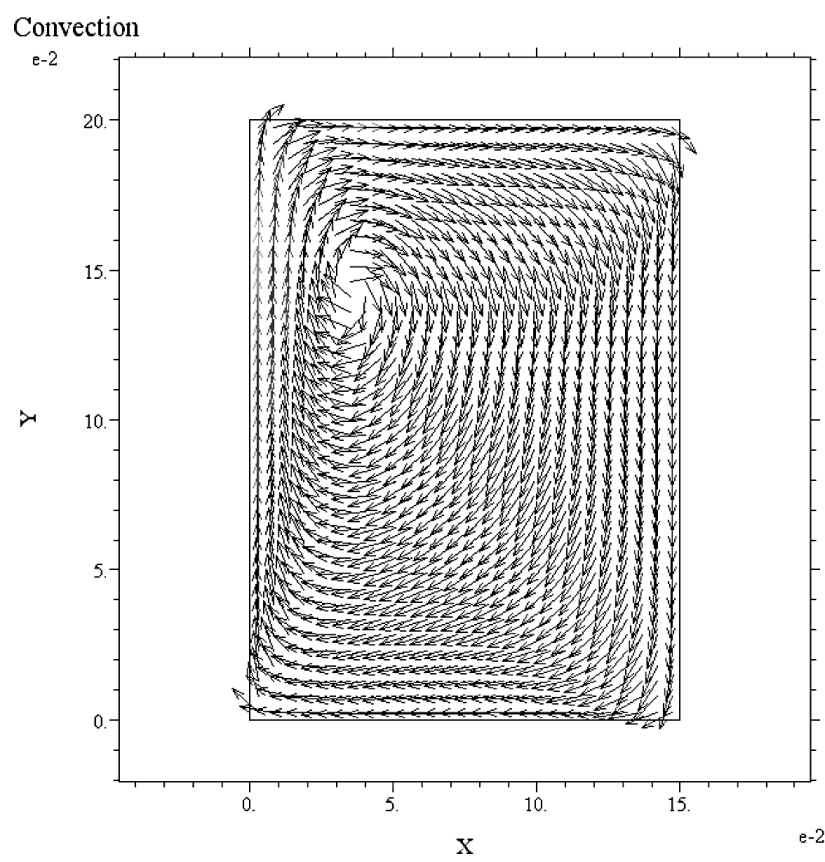

$16: 11: 262 / 27 / 13$

FlexPDE 5.0.20

Flow Velocity

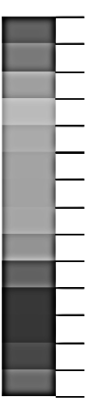

$$
\begin{aligned}
& 0.14 \\
& 0.13 \\
& 0.12 \\
& 0.11 \\
& 0.10 \\
& 0.09 \\
& 0.08 \\
& 0.07 \\
& 0.06 \\
& 0.05 \\
& 0.04 \\
& 0.03 \\
& 0.02 \\
& 0.01 \\
& 0.00
\end{aligned}
$$

Рис. 3. Результаты расчетного эксперимента: распределение скорости потока в объеме

Для двухмерного случая уравнение Навье - Стокса (1) в переменных «функция тока - вихрь скорости» имеет вид [4]

$$
\frac{\partial w}{\partial \tau}+(w \cdot \nabla) \omega=\mu \Delta \omega
$$

Система уравнений (3), (6) и (7) является искомой системой дифференциальных уравнений, используемой для задачи расчета конвекции в объеме. Полученная система уравнений решалась при помоши программного пакета Flexpde [6]. В качестве граничных условий для рассматриваемой системы уравнений использовались данные, соответствующие параметрам экспериментального стенда. Результаты расчета представлены на рис. 3 и 4.

Результаты расчета показывают, что вблизи греющей стенки наблюдается устойчивый поток жидкости, устремленный вверх. Толщина слоя в среднем составляет 0,02 м, средняя скорость подъема слоя $-0,06$ м/с (рис. 3). Движение жидкости в верхней части объема происходит в направлении «от» греющей стенки. Скорость и толщина

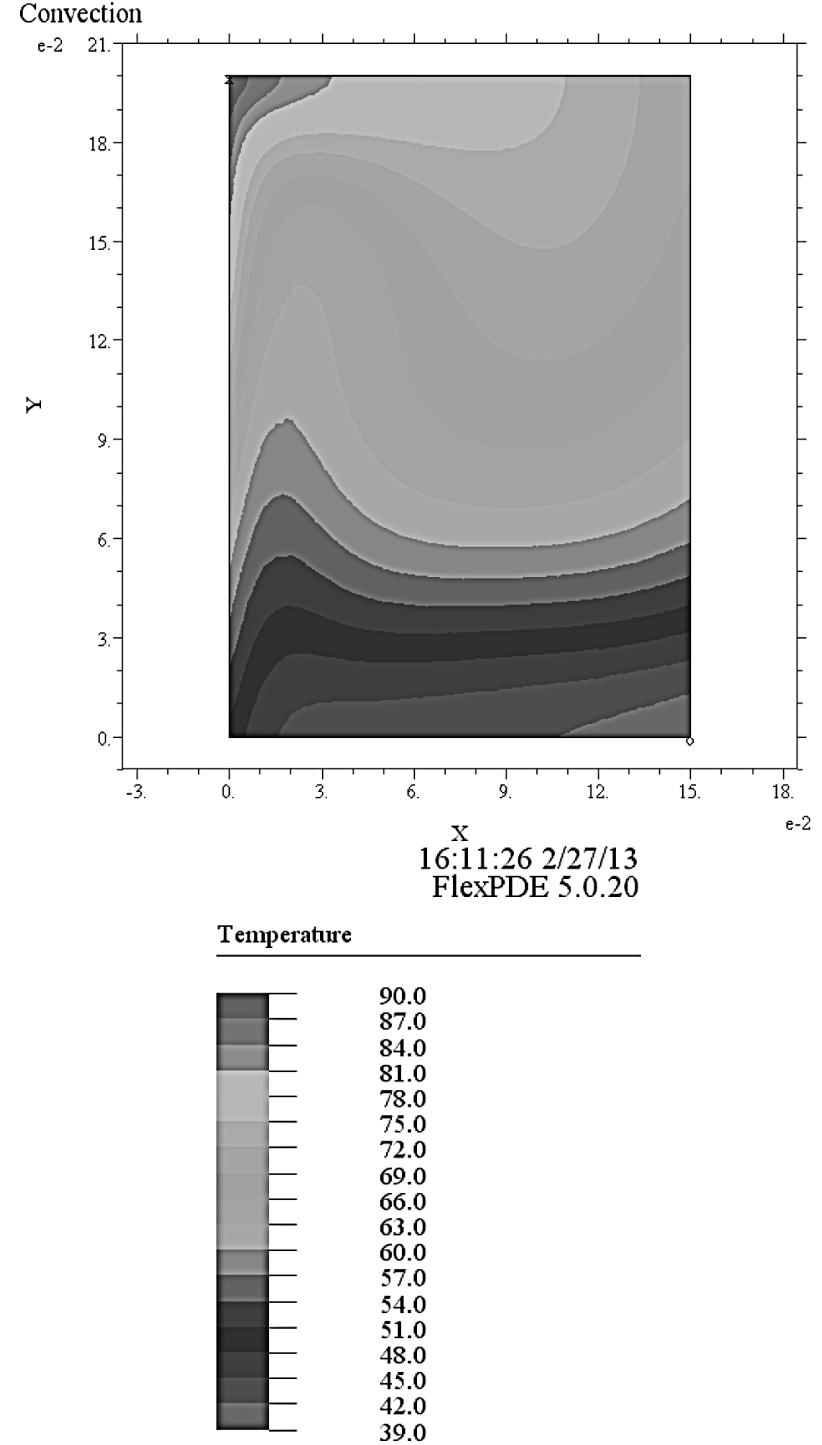

Рис. 4. Результаты расчетного эксперимента: распределение температуры в объеме

слоя жидкости, движущейся вдоль поверхности, изменяется со временем в зависимости от отдаленности потока от греющей стенки. Средняя скорость потока на поверхности составляет $0,03 \mathrm{~m} / \mathrm{c}$. Подобно результатам стендового эксперимента, начинается медленное закручивание всего нагретого потока (рис. 3). Рассогласования в полученных значениях обусловлены как некоторыми неточностями заданных граничных условий расчета, так и погрешностью измерительных приборов стендовой установки.

Расчетный эксперимент с использованием кода MELCOR. Модель экспериментального стенда, созданная с помощью кода MELCOR, состоит из шести контрольных объемов, шести путей соединения и трех тепловых структур (рис. 5), исходные параметры которых соответствуют реальным параметрам рассмотренного выше стенда.

Контрольные объемы Cv001 и Cv002 представляют собой вертикальные объемы, которые моделируют «подъемный» и «опускной» участки нагреваемой жидкости, соответственно. Контрольные объемы Cv011 и Cv012 горизонтальные объемы, моделирующие «верхний» и «нижний» участки нагреваемой жидкости. Размеры 


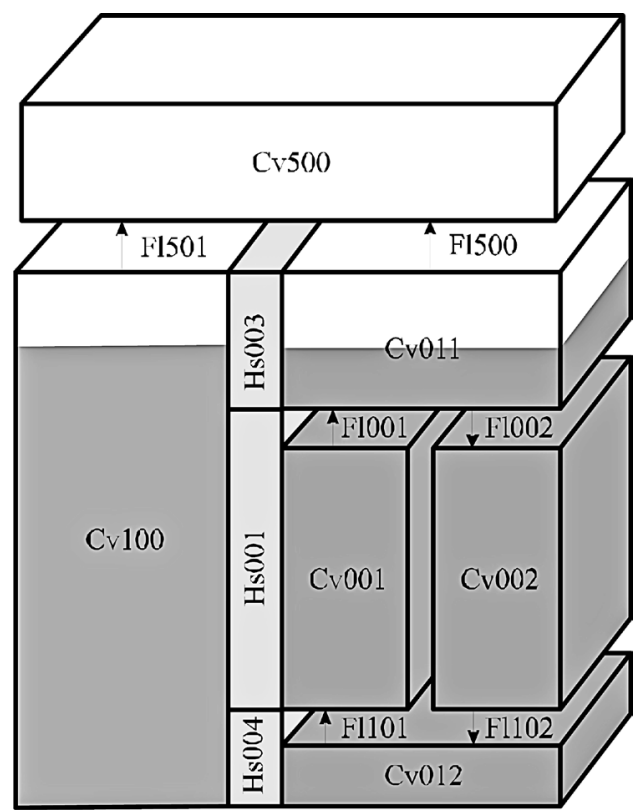

Рис. 5. Нодализационная схема установки

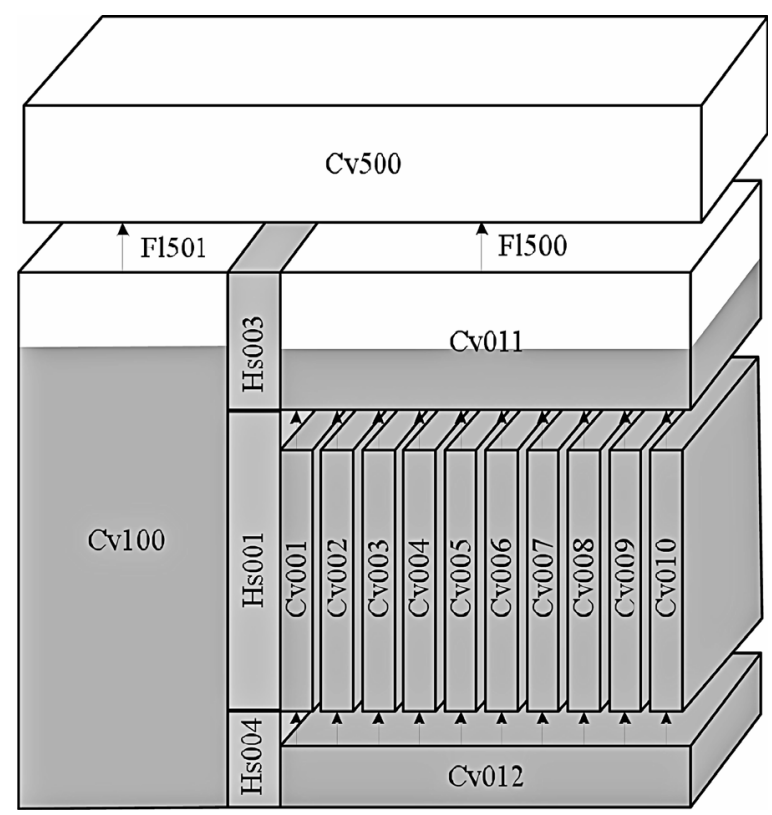

Рис. 6. Нодализационная схема установки с детальной разбивкой «подъемного» и «опускного» участков

что негативно влияет на корректность результатов расчета Было принято решение произвести более детальную нодализацию рассматриваемой установки (рис. 6).

В результате применения более детальной разбивки рассматриваемого объема скорость вблизи греющей стенки (контрольный объем Cv001) увеличилась до $0,051 \mathrm{M} / \mathrm{c}$ (рис. 7).

Так как рассогласования параметров контрольных объемов Cv002-Cv010 относительно невелики, можно принять допущение об их эквивалентности и, без влияния на результат, снизить количество расчётных объемов и пу-

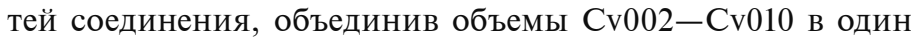
(рис. 8).

Для выяснения влияния соотношения сечений «подъемного» и «опускного» участков модели на результаты расчета были проведены эксперименты, результаты которых

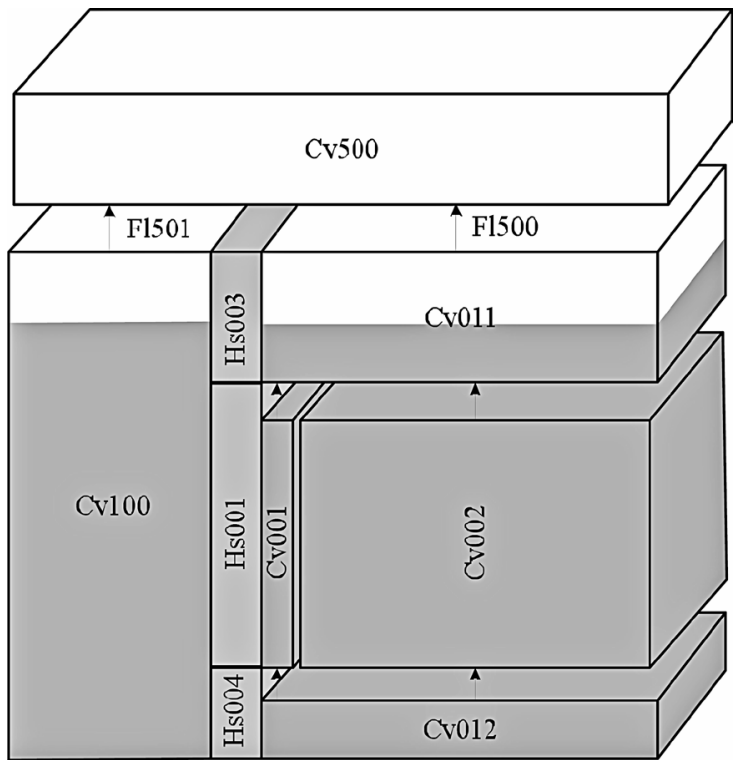

Рис. 8. Нодализационная схема установки с объединенным опускным участком 
показали, что оптимальная толщина подъемного участка находится в районе $0,02 \mathrm{M}$, что соответствует толщине подъемного участка экспериментального стенда. Аналогичным образом получена оптимальная высота верхнего контрольного объема, которая с некоторой зоной нечувствительности соответствует толщине верхнего слоя экспериментальной установки. Результаты расчета представлены на рис. 9.

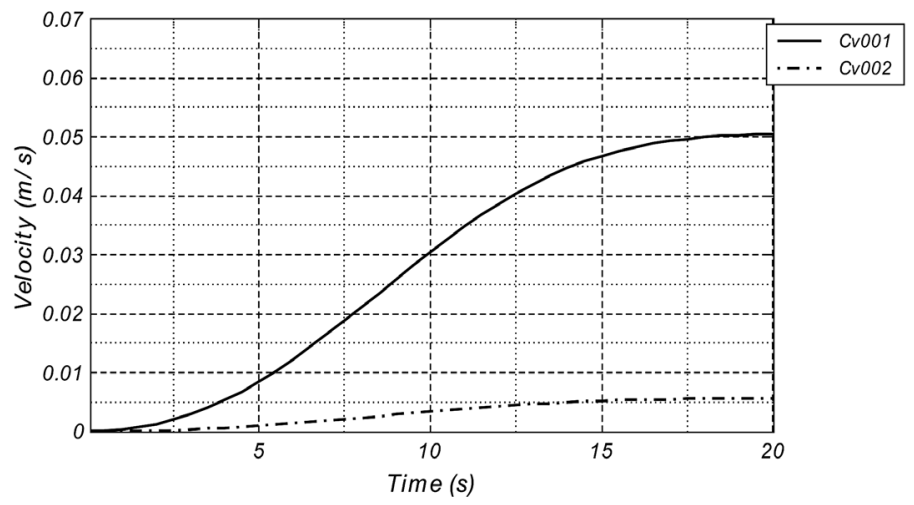

Рис. 9. Скорость движения жидкости в объемах $\mathrm{Cv001}$ и $\mathrm{Cv002}$

В результате произведенных изменений скорость на подъемном участке осталась равной 0,051 м/с. Скорость в опускном участке соответствует средней скорости необъединенных опускных объемов $(\mathrm{Cv} 002-\mathrm{Cv} 010)$ и равна 0,006 м/с. Скорость движения жидкости на верхнем участке равна $0,04 \mathrm{~m} / \mathrm{c}$.

Параметры установки, полученные экспериментальным путем, путем расчёта системы дифференциальных уравнений и при моделировании компьютерным кодом MELCOR, сведены в табл. 1.

Таблица 1. Сравнительная таблица экспериментальных и расчетных результатов

\begin{tabular}{|c|c|c|c|}
\hline Параметр & $\begin{array}{c}\text { Стендо- } \\
\text { вый эк-- } \\
\text { перимент }\end{array}$ & $\begin{array}{c}\text { Матема- } \\
\text { тическая } \\
\text { модель }\end{array}$ & $\begin{array}{c}\text { Компью- } \\
\text { терная } \\
\text { модель }\end{array}$ \\
\hline $\begin{array}{c}\text { Скорость движения } \\
\text { жидкости подъемного } \\
\text { участка, м/с }\end{array}$ & 0,05 & 0,06 & 0,051 \\
\hline $\begin{array}{c}\text { Толщина подъемного } \\
\text { участка, м }\end{array}$ & 0,019 & 0,02 & 0,02 \\
\hline $\begin{array}{c}\text { Скорость движения } \\
\text { жидкости вдоль } \\
\text { поверхности, м/с }\end{array}$ & 0,027 & 0,03 & 0,038 \\
\hline $\begin{array}{c}\text { Скорость движения } \\
\text { жидкости опускного } \\
\text { участка, м/с }\end{array}$ & 0,005 & 0,009 & 0,006 \\
\hline
\end{tabular}

Данные, приведенные в табл. 1, физически корректны и имеют достаточную сходимость. Рассогласования в значениях обусловлены:

неточностью заданных граничных условий расчетов;

погрешностью измерительных приборов стендовой установки;

использованием различных подходов для реализации расчета.
Основываясь на полученных результатах, можно утверждать, что для физически корректного моделирования конвективных систем с использованием одномерных кодов необходимо, наряду с основными параметрами системы, обладать информацией о толщине подъемного и верхнего участков. Такую информацию можно получить исходя из температуры греющей поверхности, температуры нагреваемой среды, количества теряемой теплоты и т. п.

В качестве примера реализации предложенной методики по моделированию конвективного перемешивания с использованием одномерных компьютерных кодов рассмотрим моделирование ламинарного участка бассейна с граничными условиями, эквивалентными рассматриваемому экспериментальному стенду.

Для определения параметров ламинарного подъемного участка воспользуемся формулой [8]

$$
\delta=x \cdot 3,93 \operatorname{Pr}^{-0,5}(0,952+\operatorname{Pr})^{0,25} \mathrm{Gr}^{-0,25} \text {, }
$$

где $\delta$ - ширина пограничного слоя; $x-x$-координата рассматриваемого элемента; $\mathrm{Pr}$ - число Прандтля; $\mathrm{Gr}-$ число Грасгофа.

Локальные числа Грасгофа и Прандтля определяются по выражениям [8]

$$
\mathrm{Gr}=\frac{\beta g x^{3}\left(T_{w}-T_{0}\right)}{v^{2}}
$$

где $\beta$ - коэффициент объемного расшения; $g$ - ускорение свободного падения; $v-$ кинематический коэффициент вязкости; $T_{w}-$ температура «горячей» стенки; $T_{0}-$ температура жидкости вдали от греющей стенки;

$$
\operatorname{Pr}=\frac{v}{a}
$$

где $a-$ коэффициент теплопередачи.

Определив ширину пограничного слоя, можно найти скорость свободной конвекции, т. е. скорость жидкости, движущейся вблизи греющей поверхности вверх:

$$
w_{x}=\frac{\rho_{0} g \beta}{\mu}\left(T_{w}-T_{0}\right)\left(\frac{\delta}{4} y-\frac{1}{2} y^{2}+\frac{1}{3 \delta} y^{3}-\frac{1}{12 \delta^{2}} y^{4}\right),
$$

где $\rho_{0}-$ начальная плотность жидкости; $\mu$ - динамический коэффициент вязкости; $y-y$-координата рассматриваемого элемента.

Начальным условиям: температура стенки $90{ }^{\circ} \mathrm{C}$, температура воды $20{ }^{\circ} \mathrm{C}$, высота подъемного участка 0,15 м соответствуют (рис. 10) ширина пограничного слоя 0,021 м (8) и скорость конвекции 0,052 м/с (11). При моделировании рассматриваемой системы с использованием одномерных кодов для получения физически корректных результатов необходимо производить разбиение на нодализационные элементы с учетом рассчитанных значений толщины и скорости подъемного слоя. Аналогично моделируется конвекция с турбулентным подъемным участком. Если получить расчётные значения параметров подъемного участка невозможно, следует проводить стендовый эксперимент, результаты которого, согласно предложенной методике, должны быть использованы при моделировании рассматриваемой системы. 


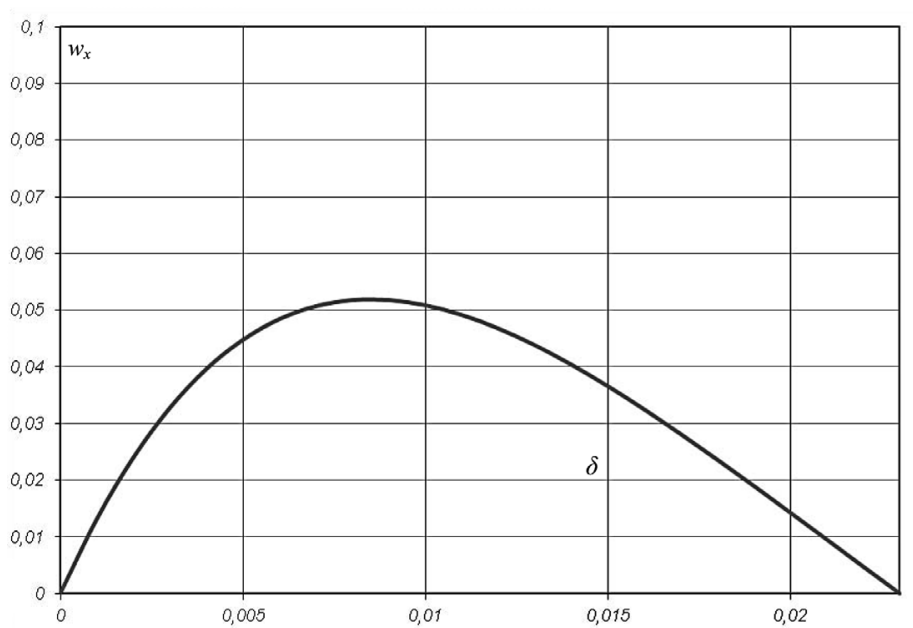

Рис. 10. Распределение скорости подъемного участка в зависимости от отдаления от греющей стенки

\section{Список использованной литературы}

1. MELCOR Computer Code Manuals. Reference Manuals. Version 1.8.5 October 2000, NUREG/CR-6119, Vol. 1, Rev. 2 SAND20002417/1, Sandia National Laboratories, Albuquerque, NM 87185-0739.

2. Ландау Л. Д., Лифшии Е. М. Гидродинамика. Теоретическая физика. - T. VI. - 3-е изд., перераб. - М.: Наука, 1986. -736 с.

3. http://www.fast-const.ru/articles.php?article_id $=21$

4. Никонов В. В. Развитие вихревых методов расчета обтекания тел несжимаемыми невязким и вязким потоками: дис. ... канд. техн. наук: 01.02.05 - механика жидкости, газа и плазмы / Никонов Валерий Владимирович. - Самара, 2007. - 174 с.

5. Самарский A. А. Вычислительная теплопередача / А. А. Самарский, П. Н. Вабищевич. - М.: Едиториал УРСС, 2003. - 784 с.

6. FlexPDE_User Guide_Version 2.19_10/30/00_Copyright (2000 PDE Solutions Inc

7. Григорьев Ю. Н. Метод вихрей в ячейках для плоских течений сжимаемых, неоднородных и релаксирующих газов / Ю. Н. Григорьев; Ин-т вычислительных технологий (Новосибирск) // Всерос. конф. по вычислительной математике КВМ2007. 18-20 июня 2007 г. : Тезисы докладов : Численное решение диф. и интеграл. уравнений. - Новосибирск: Ин-т вычислительной математики и математической геофизики СОРАН. - 2007.

8. Дульнев Г. Н. Теория тепло- и массообмена / Г. Н. Дульнев. - СПб: НИУ ИТМО, 2012. - 195 с.

\section{References}

1. MELCOR Computer Code Manuals. Reference Manuals. Version 1.8.5 October 2000, NUREG/CR-6119, Vol. 1, Rev. 2 SAND20002417/1, Sandia National Laboratories, Albuquerque, NM 87185-0739.

2. L. Landau, E. Lifshitz. Hydrodynamics. Theoretical physics. V. VI - 3rd publication. - M.: Science, 1986. - 736 p.) (Rus)

3. http://www.fast-const.ru/articles.php?article_id $=214$.

4. Nikonov $V$.. Development of methods for calculating the vortex flow of bodies and non-viscous incompressible viscous flows: Dis. Sciences: 01.02.05 - Mechanics of liquid, gas and plasma / Valery Nikonov. - Samara, 2007. - 174 p. (Rus)

5. Samarsky A. Computational Heat Transfer / A. Samarsky, P. Vabishchevich. - Moscow: Editorial URSS, 2003. - 784. (Rus)

6. FlexPDE_User Guide _Version 2.19_10/30/00_Copyright (C) 2000 PDE Solutions Inc.

7. Grigoryev $Y u$. Method of vortices in the cells for plane flows of compressible, non-uniform and relaxing gases / Yu. Grigoryev, Institute of Computing Technology (Novosibirsk) / Conf. on Computational Mathematics ICCM-2007. 18-20 June 2007: Abstracts: The numerical solution of differential. and integral. equations. - Novosibirsk: Institute of Computational Mathematics and Mathematical Geophysics SB RAS. - 2007. (Rus)

8. Dulnev G. Theory of Heat and Mass Transfer / G. Dulnev. St. Petersburg: ITMO, 2012. -195 p. (Rus)

Получено 16.07.2013. 